\title{
Hysteresis in a Rotating Differentially Heated Spherical Shell of Boussinesq Fluid*
}

\author{
Gregory M. LeWIS \\ Faculty of Science \\ University of Ontario Institute of Technology \\ Oshawa ON L1H 7K4, Canada \\ greg.lewis@uoit.ca \\ WiLLIAM F. LANGFORD \\ Department of Mathematics and Statistics \\ University of Guelph \\ Guelph ON N1G 2W1, Canada \\ wlangfor@uoguelph.ca
}

\begin{abstract}
A mathematical model of convection of a Boussinesq fluid in a rotating spherical shell is analyzed using numerical computations guided by bifurcation theory. The fluid is differentially heated on its inner spherical surface, with the temperature increasing from both poles to a maximum at the equator. The model is assumed to be both rotationally symmetric about the polar axis and reflectionally symmetric across the equator. This work is an extension to spherical geometry of previous work on the differentially heated rotating annulus. The spherical geometry is motivated by applications to planetary atmospheres. As the temperature gradient increases from zero, large Hadley cells extending from equator to poles form immediately. For larger temperature differences, two or three convection cells appear in each hemisphere. An organizing centre is shown to exist, at which two saddle-node bifurcations come together in a codimension-2 hysteresis bifurcation (or cusp) point, providing a mechanism for hysteretic transitions between different cell patterns as the temperature gradient is varied.
\end{abstract}

Keywords: cusp point, hysteresis bifurcation, flow transitions, numerical computation, large-scale geophysical flow

AMS Subject headings: 37N10, 76U05, 37M99

${ }^{*}$ This work was supported in part by the Natural Sciences and Engineering Research Council of Canada and by the Fields Institute for Research in Mathematical Sciences. 


\section{Introduction}

The atmosphere of a planet may be idealized as a spherical shell of fluid surrounding the spherical surface of the planet. Many factors affect the circulation of the atmosphere; chief among these are the rotation of the planet, the differential heating of the surface and atmosphere by the planet's sun, and the thickness and composition of the atmosphere itself. In this paper we construct an idealized mathematical model of such a planet, ignoring all local variations of surface features such as oceans, continents, mountains and glaciers. See Figure 1 . The symmetries of this model are exploited to make the computations more tractable.

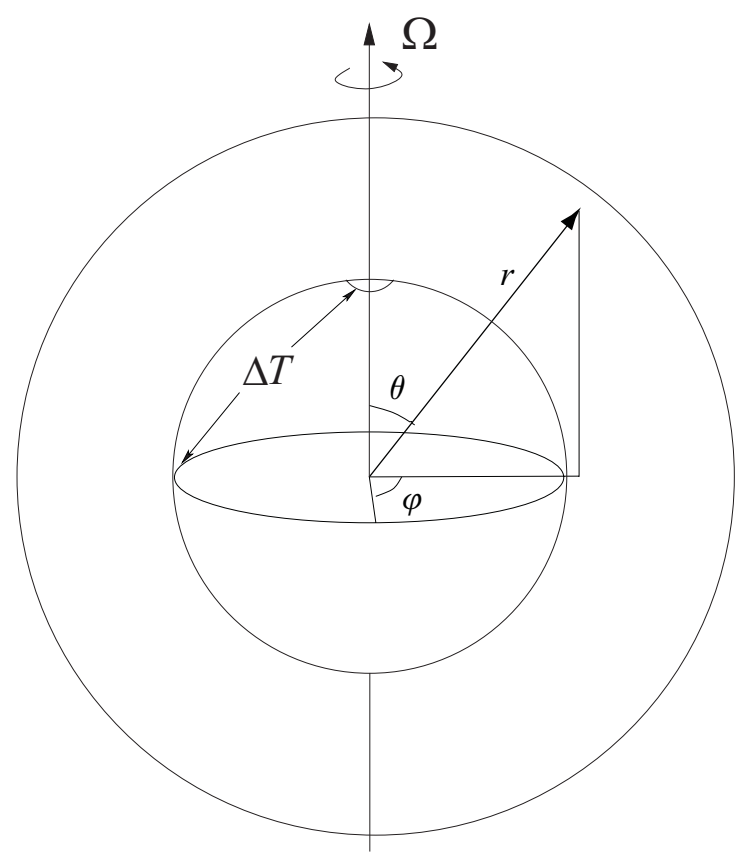

Figure 1: A differentially heated rotating spherical shell rotated at rate $\Omega$. The inner sphere is held at the temperature $T=T_{r}-\Delta T \cos (2 \theta)$, creating a differential heating between the equator and the pole (see text). Polar spherical coordinates are given by $(r, \theta, \varphi)$ as shown.

On the inner boundary surface a temperature profile is prescribed to reflect the differential heating of the atmosphere by the sun, as follows. The average annual flux of solar radiation on a planet whose axis of rotation is tilted approximately $20^{\circ}$ with respect to the plane that is perpendicular to the solar rays is nearly proportional to $-\cos (2 \theta)$, see [18], where $\theta$ is the polar angle, which only differs from the latitude in its range (in particular the latitude is given by $\pi / 2-\theta)$. This flux is independent of the azimuthal variable $\varphi$ and is a maximum at the equator $(\theta=\pi / 2)$, and a minimum at the poles $(\theta=0, \pi)$. This profile is similar to the annual average of solar flux for many of the planets in our solar system, including Earth. Therefore, we choose the temperature on the inner boundary surface to be fixed at $T=T_{r}-\Delta T \cos (2 \theta)$, where $T_{r}$ is a reference temperature and the difference 
in the temperature from equator to pole is $2 \Delta T$, see Figure 2. On a real planet, a flux of radiation of this form would not result exactly in this prescribed temperature distribution on the surface, but we expect it to capture the gross effects of the differential heating. In the model, the spherical shell is filled with a Boussinesq fluid, which means that its density varies linearly with temperature. Then the gravitational force acting radially inward drives convective motions of the fluid when $\Delta T>0$.

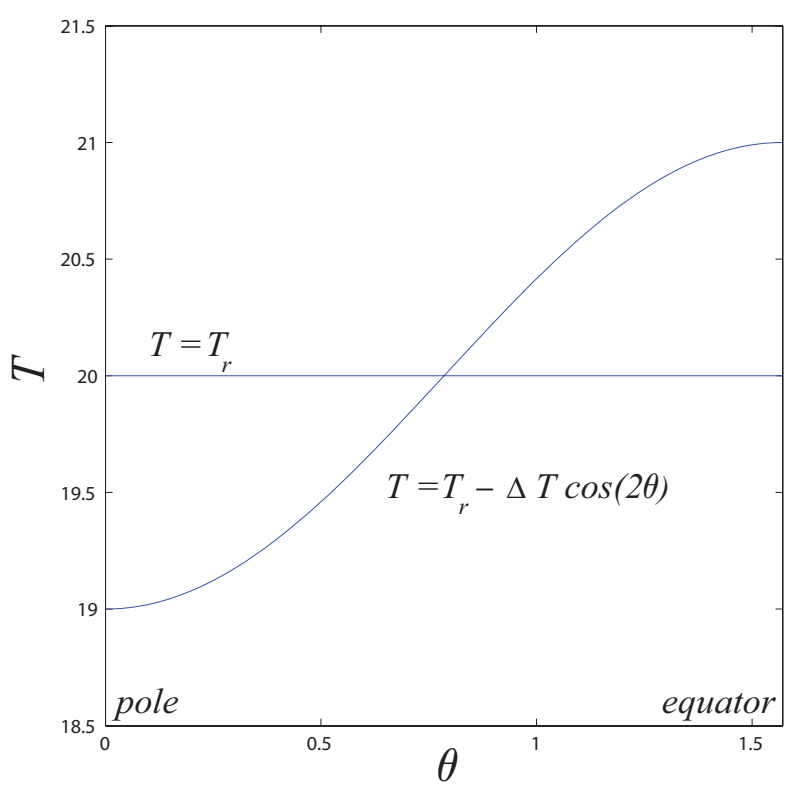

Figure 2: Differential temperature profile on the inner boundary, proportional to the solar energy flux on the surface of a tilted rotating planet (case $\Delta T=1, T_{0}=20$ ).

The fluid flow is assumed to satisfy non-slip boundary conditions on the inner sphere and stress-free boundary conditions on the outer sphere. We assume that the outer sphere of the shell is rigid and thermally insulating, which implies that there is no flow of fluid or heat across the outer boundary. Inside the spherical shell, the fluid satisfies the Navier-Stokes equations in the Boussinesq approximation. Complete details of the model are given in Section 2. The nonlinear equations for a steady state are solved using Newton iteration with Keller continuation from the trivial solution at $\Delta T=0$. The linear stability problem is solved using the implicitly restarted Arnoldi method following a generalized Cayley transformation. The full methodology for analysis of the model is described in Sections 3 and 4 and the results are presented in Sections 5 and 6.

In 1735, George Hadley published an explanation of the trade winds, which exist in a band approximately $30^{\circ}$ to either side of the equator, blowing very steadily from the northeast in the northern hemisphere and from the southeast in the southern hemisphere [10, 22]. He argued that differential heating causes the warmer air over the equator to rise, drawing 
cooler air from north and south towards the equator, near the surface of the Earth. The rising warm air cools and then moves away from the equator to the north and south at high altitudes. Eventually, it descends back to the surface at latitudes of approximately $\pm 30^{\circ}$ from the equator and completes the loop, warming and moving back towards the equator near the surface. He explained the strong westward component of the trade winds as being due to what is now known as the Coriolis force, caused by rotation of the Earth. (Coriolis' work was to appear a century later.) These two large toric vortices ringing the Earth near the equator are now called Hadley cells. In reality they are not always centered about the equator, but undergo an annual north-south migration following the sun.

It is not possible in an Earth-bound laboratory to perform an experimental study of convection in a spherical shell as shown in Figure 1, because the gravitational force can not be directed radially towards the common center of the spheres. (However, experiments to study spherical convection with a central force field under weightless conditions in a space lab are an interesting possibility, see for example $[11,2]$.) Many laboratory experiments have been performed on a differentially heated rotating cylindrical annulus, with the Earth's gravity acting downward parallel to the cylinder axis and centrifugal force acting radially. These experiments typically used water as the working fluid. Much has been learned about the dynamics of large scale geophysical fluids from these laboratory experiments, even though the Reynolds number is significantly smaller in the experimental fluids than in actual geophysical flows $[12,22]$. The Boussinesq approximation has been used as a basis for a mathematical model of the differentially heated rotating annulus, using laboratory-scale parameters and with water as the fluid $[13,19,20]$. The model has provided good agreement with the corresponding laboratory experiments.

Therefore, in this prototype mathematical model of a differentially heated rotating spherical shell of fluid, we adopt a philosophy of choosing the parameters of the fluid to be those of water and using the laboratory scale rather than the geophysical. In this way, the computations are tractable and the results obtained can be compared to both experiments and theoretical calculations for the better understood case of a differentially heated rotating annulus. This may provide insight into the how the geometry of the system leads to the observed flow transitions, and may serve as a step towards understanding the dynamical structure of planetary systems. Although we have made quantitative choices for the fluid and the boundary conditions, we expect that these choices will have little effect on qualitative features of the results. This is supported by the numerous studies that have been performed on the differentially heated rotating annulus. Many experiments have been performed with different forms of heating, different geometries of the apparatus and with different fluid properties, and it is found that these have little qualitative effect on the types of transitions and 
form of the transition curves that are observed.

The goal of this paper is to determine the flow patterns, their stabilities and their transitions (bifurcations) for a differentially heated rotating spherical shell, consistent with our modeling assumptions. There are three bifurcation parameters of interest: the temperature difference $\Delta T$, the shell gap width $R$ and the rotation rate $\Omega$. The results presented in Section 5 show that the qualitative features of the flow patterns do not change for moderate changes of $\Omega$, although the stability with respect to non-rotationally symmetric perturbations is affected. However, for even very small $\Delta T>0$, the fluid immediately begins to flow in a large stable convection cell with easterly flow toward the equator near the inner surface, similar to a Hadley cell except that it extends from equator to pole (in each hemisphere). In this paper, we refer to this as the Hadley cell. For larger values of $\Delta T$, two or even three convection cells may appear between the equator and pole. The Hadley cell shrinks, always keeping one edge at the equator, while the additional cells appear between it and the pole. When a second cell exists next to the Hadley cell, it is characterized by a weaker counter-rotating flow, with a westerly component. The third cell, when it exists, has the same rotation as the Hadley cell but lies near the pole. In every case, there is a region of high velocity azimuthal flow at high altitudes and mid-latitudes, resembling the Earth's jet stream.

Furthermore, we show that there exists a critical value of the pair $(R, \Delta T)$ that is a hysteresis bifurcation point (also called a cusp point), see Figure 3. This is a codimension-2 steady-state bifurcation that is well understood in mathematical bifurcation theory. The existence of this hysteresis point is demonstrated by an explicit calculation of its defining conditions in Section 6, where the study of this hysteresis bifurcation is presented in detail. Only its main features are outlined here. In Figure 3, $M$ is a manifold of steady states, existing for given parameters $(R, \Delta T)$, and the vertical axis represents the amplitude of a steady state (determined by the stream function in Section 6). In a neighborhood of a hysteresis bifurcation point, the number of steady states (on $M$ ) can vary from one to three as the parameters $(R, \Delta T)$ vary. When three steady states coexist in Figure 3 , the middle one is unstable, but all other steady states in Figure 3 remain stable (attracting). Furthermore, there are abrupt upward and downward transitions at two fold bifurcations (also called limit points or saddle-node bifurcations) as shown in Figure 3, that occur at both ends of an interval of the bifurcation parameter $\Delta T$, for appropriate fixed $R$. If $\Delta T$ is varied back and forth through this interval, the system traces a hysteresis loop as shown in Figure 3. Between these two abrupt transitions there is an interval of $\Delta T$ that exhibits bistability; that is, two solutions are stable simultaneously. 


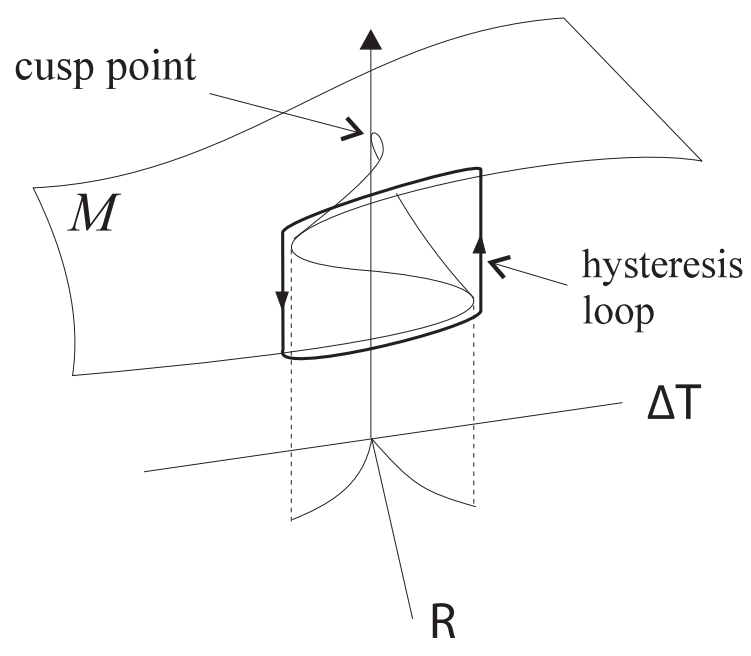

Figure 3: The codimension 2 hysteresis bifurcation, showing the hysteresis loop as $\Delta T$ varies back and forth. The cusp shown in the $(R, \Delta T)$ parameter plane is the projection of the two curves of fold bifurcations onto this plane.

\section{$1.1 \quad$ Relationship to previous work}

The fundamental problem of convection in spherical shells was formulated by Chandrasekhar [4, Ch.6], who also solved the stability problem in the Boussinesq approximation for the spherically symmetric case, in terms of spherical harmonics. There is a large literature on spherically symmetric convection that is motivated by the Earth's core and mantle, where the boundary conditions have full spherical symmetry (in particular a constant temperature on the inner sphere), see for example $[2,5,21,32]$. The present work differs from all of these because of the latitudinal temperature gradient.

Motivation for the present work is provided also by the classical Taylor-Couette experiment, in which the flow of a fluid (usually water) between two differentially rotating long coaxial cylinders is studied, see for example $[1,6,8,16]$. In that experiment many interesting flow patterns may form, including Taylor vortices, which are invariant tori stacked coaxially between the cylinders in pairs with alternating clockwise and counterclockwise helical flows. There is no differential heating in the Taylor-Couette experiment; even so, the Taylor vortices strongly resemble Hadley cells. Marcus and Tuckerman [23, 24] performed numerical simulations of the flow between two differentially rotating spheres (without heating) and found bifurcations to different numbers of cells that they called Taylor vortices, thus showing that Hadley-like cells may form even without differential heating, if the spheres are rotated differentially. 


\section{Model equations}

We use the Navier-Stokes equations in the Boussinesq approximation to model the fluid flow within the spherical shell. In the Boussinesq approximation, the variations of all fluid properties, except the density, are considered to be negligible and the equation of state of the fluid is assumed to be

$$
\rho=\rho_{0}\left(1-\alpha\left(T-T_{r}\right)\right)
$$

where $\rho$ is the density of the fluid, $T$ is the temperature, $\alpha$ is the (constant) coefficient of thermal expansion, and $\rho_{0}$ is the density at a reference temperature $T_{r}$. The dimensionless quantity $\alpha\left(T-T_{r}\right)$ is assumed to be small. In the Boussinesq approximation the fluid can be considered to be incompressible, which is a significant simplification.

The fluid is contained within a spherical shell with inner sphere of radius $r_{a}$ and outer sphere of radius $r_{b}$. We assume gravity acts everywhere in the radial direction. The spherical shell rotates at rate $\Omega$ about the polar axis, and the inner and outer spheres rotate at the same rate. The equations are written in spherical polar coordinates in a frame of reference co-rotating at rate $\Omega$ with the shell. The radial, polar, and azimuthal coordinates are denoted $r, \theta$, and $\varphi$, respectively, with unit vectors $\mathbf{e}_{r}, \mathbf{e}_{\theta}$ and $\mathbf{e}_{\varphi} ;$ see Figure 1.

The Navier-Stokes Boussinesq equations describing the evolution of the vector fluid velocity, $\mathbf{u}=\mathbf{u}(r, \theta, \varphi, t)=w \mathbf{e}_{r}+v \mathbf{e}_{\theta}+u \mathbf{e}_{\varphi}$ and the temperature of the fluid, $T=T(r, \theta, \varphi, t)$ are:

$$
\begin{aligned}
\frac{\partial \mathbf{u}}{\partial t}= & \nu \nabla^{2} \mathbf{u}-2 \boldsymbol{\Omega} \times \mathbf{u}+\left[g \mathbf{e}_{r}+\boldsymbol{\Omega} \times(\boldsymbol{\Omega} \times \mathbf{r})\right] \alpha\left(T-T_{r}\right)-\frac{1}{\rho_{0}} \nabla p-(\mathbf{u} \cdot \nabla) \mathbf{u}, \\
\frac{\partial T}{\partial t}= & \kappa \nabla^{2} T-(\mathbf{u} \cdot \nabla) T, \\
& \nabla \cdot \mathbf{u}=0
\end{aligned}
$$

where $\Omega=\Omega\left(\cos \theta \mathbf{e}_{r}-\sin \theta \mathbf{e}_{\theta}\right)$ is the rotation vector, $\Omega=|\Omega|$ is the rate of rotation about the polar axis, $p$ is the pressure deviation from $p_{0}=\rho_{0} g(R-r)+\rho_{0} \Omega^{2} r^{2} \sin ^{2} \theta / 2$, $\mathbf{r}=r \mathbf{e}_{r}+\theta \mathbf{e}_{\theta}+\varphi \mathbf{e}_{\varphi}, \nu$ is the kinematic viscosity, $\kappa$ is the coefficient of thermal diffusivity, $g$ is the gravitational acceleration, $\nabla$ is the usual gradient operator in spherical coordinates, $u$ is the azimuthal fluid velocity, often referred to as the zonal velocity, $v$ is the polar fluid velocity, and $w$ is the radial fluid velocity. The spatial domain is defined by $r_{a}<r<r_{b}$, $0 \leq \varphi<2 \pi$, and $0<\theta<\pi$. Thus, $\theta=0, \pi$ correspond to the north and south poles of the shell respectively, while $\theta=\pi / 2$ corresponds to the equator. The equations can be rewritten in planetary coordinates by performing the change of variable $\theta \rightarrow \pi / 2-\theta$. The values of $\nu$ and $\kappa$ are chosen to be those of the fluid at the reference temperature $T_{r}$, and it is assumed that the difference between the temperature of the fluid and $T_{r}$ is everywhere small enough so that $\nu$ and $\kappa$ can be considered as constants. We have included the effects of 
centrifugal buoyancy in the equations via the term $\Omega \times(\boldsymbol{\Omega} \times \mathbf{r})$. All dimensional quantities are measured in CGS units.

As described in the Introduction, the boundary conditions are

$$
\begin{gathered}
\mathbf{u}=0, \quad T=T_{r}-\Delta T \cos (2 \theta) \quad \text { on } \quad r=r_{a}, \\
\frac{\partial u}{\partial r}=0, \quad \frac{\partial v}{\partial r}=0, \quad w=0, \quad \frac{\partial T}{\partial r}=0 \quad \text { on } \quad r=r_{b},
\end{gathered}
$$

with $2 \pi$-periodicity in the azimuthal variable $\varphi$.

In this paper we will investigate flows that preserve the symmetries of the model, that is, flows that are invariant under rotation about the polar axis (i.e. they are independent of the azimuthal variable $\varphi$ ), and invariant under reflection across the equator (i.e. across the line defined by $\theta=\pi / 2)$. Therefore, we study solutions of $(2)-(5)$ in the form

$$
\begin{aligned}
u=u(r, \theta, t) & =u(r, \pi-\theta, t), & v & =v(r, \theta, t)=v(r, \pi-\theta, t), \\
w & =w(r, \theta, t)=w(r, \pi-\theta, t), & T & =T(r, \theta, t)=T(r, \pi-\theta, t),
\end{aligned}
$$

In this context, solutions that are independent of $\varphi$ are often called axisymmetric. The assumed symmetries significantly simplify the analysis. We may use the analysis of the symmetric system as a starting point for an analysis of the full system. Although it is not written explicitly, the solutions also depend on the parameters.

If we scale the radial coordinate as

$$
r \rightarrow R r^{\prime}
$$

where $R=r_{b}-r_{a}$ is the gap width, write the temperature as

$$
T \rightarrow T^{\prime}+T_{r}-\Delta T \cos (2 \pi)
$$

substitute these into (2) - (4), and drop the primes, we obtain the following equations describing the evolution of the fluid velocity $\mathbf{u}=w(r, \theta, t) \mathbf{e}_{r}+v(r, \theta, t) \mathbf{e}_{\theta}+u(r, \theta, t) \mathbf{e}_{\varphi}$, 
pressure deviation $p=p(r, \theta, t)$ and temperature deviation $T=T(r, \theta, t)$ :

$$
\begin{gathered}
\frac{\partial u}{\partial t}=\nu_{s} \nabla_{0}^{2} u-\nu_{s} \frac{1}{r^{2} \sin ^{2} \theta} u-2 \Omega(\sin \theta w+\cos \theta v)-\frac{1}{R}\left[\left(\mathbf{u} \cdot \nabla_{0}\right) u+\frac{\cos \theta}{r \sin \theta} u v+\frac{u w}{r}\right] \\
\frac{\partial v}{\partial t}=\nu_{s} \nabla_{0}^{2} v-\nu_{s}\left(\frac{1}{r^{2} \sin ^{2} \theta} v-\frac{2}{r^{2}} \frac{\partial w}{\partial \theta}\right)+2 \Omega \cos \theta u-\frac{1}{\rho_{0} R r} \frac{\partial p}{\partial \theta} \\
\quad-\left(\alpha \Omega^{2} R r \sin \theta \cos \theta\right)(T-\Delta T \cos 2 \theta)-\frac{1}{R}\left[\left(\mathbf{u} \cdot \nabla_{0}\right) v-\frac{\cos \theta}{r \sin \theta} u^{2}+\frac{v w}{r}\right] \\
\frac{\partial w}{\partial t}=\nu_{s} \nabla_{0}^{2} w-\nu_{s}\left(\frac{2}{r^{2}} \frac{\cos \theta}{\sin \theta} v+\frac{2}{r^{2}} \frac{\partial v}{\partial \theta}+\frac{2}{r^{2}} w\right)+2 \Omega \sin \theta u-\frac{1}{\rho_{0} R r} \frac{\partial p}{\partial r} \\
\quad-\alpha\left(\Omega^{2} R r \sin ^{2} \theta+g\right)(T-\Delta T \cos 2 \theta)-\frac{1}{R}\left[\left(\mathbf{u} \cdot \nabla_{0}\right) w-\frac{1}{r}\left(u^{2}+v^{2}\right)\right] \\
\frac{\partial T}{\partial t}=\kappa_{s} \nabla_{0}^{2} T+\frac{4 \Delta T \kappa_{s}}{r^{2}}\left(\cos 2 \theta+\cos ^{2} \theta\right)+\frac{2 \Delta T}{R r} \sin 2 \theta v-\frac{1}{R}\left(\mathbf{u} \cdot \nabla_{0}\right) T \\
\nabla_{0} \cdot \mathbf{u}=\frac{\partial w}{\partial r}+\frac{2}{r} w+\frac{1}{r} \frac{\partial v}{\partial \theta}+\frac{\cos \theta}{r \sin \theta} v=0
\end{gathered}
$$

where $\nu_{s}=\nu / R^{2}, \kappa_{s}=\kappa / R^{2}$,

$$
\begin{gathered}
\nabla_{0}^{2}=\frac{\partial^{2}}{\partial r^{2}}+\frac{2}{r} \frac{\partial}{\partial r}+\frac{1}{r^{2}} \frac{\partial^{2}}{\partial \theta^{2}}+\frac{\cos \theta}{r^{2} \sin \theta} \frac{\partial}{\partial \theta} \\
\nabla_{0}=\mathbf{e}_{r} \frac{\partial}{\partial r}+\mathbf{e}_{\theta} \frac{1}{r} \frac{\partial}{\partial \theta}
\end{gathered}
$$

and

$$
\left(\mathbf{u} \cdot \nabla_{0}\right) f=w \frac{\partial f}{\partial r}+\frac{v}{r} \frac{\partial f}{\partial \theta}
$$

for any scalar function $f=f(r, \theta, t)$. The domain is now expressed as $r_{a} / R<r<r_{b} / R$, $0 \leq \theta<\pi / 2$, and the boundary conditions become

$$
\begin{array}{ccc}
\mathbf{u} & =0, \quad T=0 \quad & \text { on } \quad r=\frac{r_{a}}{R}, \\
w=0, & \frac{\partial u}{\partial r}=\frac{\partial v}{\partial r}=0 \quad \frac{\partial T}{\partial r}=0 & \text { on } \quad r=\frac{r_{b}}{R} .
\end{array}
$$

The symmetry assumptions not only reduce the domain size, i.e. we now have $0 \leq \theta \leq$ $\pi / 2$, but they also effectively introduce new boundary conditions at the equator and the pole. In order to satisfy the symmetries, there can be no flow of fluid or heat across the equator or the pole. Thus, we have the additional boundary conditions:

$$
\begin{aligned}
& u, v=0, \quad \frac{\partial w}{\partial \theta}=0, \quad \frac{\partial T}{\partial \theta}=0 \quad \text { on } \quad \theta=0, \\
& v=0, \quad \frac{\partial u}{\partial \theta}=\frac{\partial w}{\partial \theta}=0, \quad \frac{\partial T}{\partial \theta}=0 \quad \text { on } \quad \theta=\pi / 2 \text {. }
\end{aligned}
$$

The condition $u=0$ at the pole is necessary to ensure that no discontinuity occurs in the fluid velocity at the pole. 
It is possible to write the equations completely in terms of dimensionless variables. However, this would not simplify the analysis, so we choose to work with the equations in the form $(9)-(13)$. This follows previous numerical work on similar problems, e.g. [13, 31].

\section{Analysis}

\subsection{Nonlinear equations for steady solution}

The analysis begins with the computation of steady axisymmetric solutions that are invariant with respect to reflection across the equator; that is, we seek solutions of $(9)-(13)$ that satisfy the boundary conditions $(15)$ - (16) and are independent of time.

The method of stream functions is used to solve the steady equations. If $v$ and $w$ are written in terms of a stream function $\xi$, defined by

$$
v=-\frac{1}{r} \frac{\partial(r \xi)}{\partial r}, \quad w=\frac{1}{r \sin \theta} \frac{\partial(\sin (\theta) \xi)}{\partial \theta},
$$

then the incompressibility condition (13) is automatically satisfied. After using (17) to replace $v$ and $w$ in the equations, the pressure terms can be eliminated. Subsequently, the steady solution can be found from the resulting three equations in the three unknown functions $u, \xi$ and $T$. These equations are found using the Maple symbolic computation software package, and are sufficiently complicated that no insight is gained by explicitly writing them here. The boundary conditions for $u$ and $T$ are given by (15) - (16) as before, while the conditions on $v$ and $w$ will be satisfied if $\xi$ satisfies the boundary conditions

$$
\begin{array}{lccc}
\xi=0, & \frac{\partial^{2} \xi}{\partial \theta^{2}}=0 & \text { on } & \theta=0, \frac{\pi}{2} \\
\xi=0, & \frac{\partial \xi}{\partial r}=0 & \text { on } & r=\frac{r_{a}}{R}, \\
\xi=0, & \frac{\partial^{2} \xi}{\partial r^{2}}+\frac{R}{r_{b}} \frac{\partial \xi}{\partial r}=0 & \text { on } & r=\frac{r_{b}}{R} .
\end{array}
$$

The numerical algorithm to solve this system of nonlinear equations is described in Section 4 and the steady axisymmetric solutions obtained are presented in Section 5.

\subsection{Linear stability analysis}

The linear stability of a steady solution is defined in terms of the eigenvalues of the linearization of the dynamical equations, about that solution. If the real parts of all the eigenvalues are negative, then all perturbations from the steady solution will decay in the linearized equations. In this case, the solution is said to be linearly stable. If any of the eigenvalues has positive real part, then some small perturbations will grow, and the solution is linearly 
unstable. If there exist only eigenvalues with zero real part and negative real part, the solution is called neutrally stable. If the real part of an eigenvalue crosses the imaginary axis as a parameter is varied, then a qualitative change in the solution occurs; i.e., a bifurcation takes place. Flows corresponding to linearly stable steady solutions can be observed physically if the noise that is naturally present is sufficiently small. If a steady solution is linearly unstable, then the corresponding flow can not be observed because some small perturbations due to the noise will tend to grow. Thus, it is expected that bifurcations correspond to transitions in the observed flow.

We compute the steady axisymmetric solution and its linear stability as the parameters of the system change, and seek locations in the space of parameters where an eigenvalue crosses the imaginary axis. We are interested in computing solutions that do not break the assumed symmetry, and therefore, we require that this eigenvalue be associated with an eigenfunction that respects the symmetry.

If we write

$$
u=u^{\prime}+u_{0}, \quad \xi=\xi^{\prime}+\xi_{0}, \quad T=T^{\prime}+T_{0},
$$

where $u_{0}, \xi_{0}, T_{0}$ is a steady solution, and substitute into the three equations for $u$, $\xi$, and $T$, we obtain the perturbation equations in $u^{\prime}, \xi^{\prime}$, and $T^{\prime}$. The trivial solution satisfies the perturbation equations, and it corresponds to $u_{0}, \xi_{0}, T_{0}$. If the perturbation equations are linearized and we assume that the unknown functions may be written as

$$
u^{\prime}(r, \theta, t)=e^{\lambda t} \psi_{u}(r, \theta), \quad \xi^{\prime}(r, \theta, t)=e^{\lambda t} \psi_{\xi}(r, \theta), \quad T^{\prime}(r, \theta, t)=e^{\lambda t} \psi_{T}(r, \theta)
$$

then a linear eigenvalue problem is obtained. Consequently, the eigenvalues $\lambda$ can be found from the generalized eigenvalue problem of the form

$$
\lambda \mathbf{A}_{0} \Psi=\mathbf{L}_{0} \Psi
$$

where

$$
\Psi=\left(\begin{array}{c}
\psi_{u} \\
\psi_{\xi} \\
\psi_{T},
\end{array}\right)
$$

is the eigenfunction, and $\mathbf{A}_{0}$ and $\mathbf{L}_{0}$ are $3 \times 3$ matrices of linear differential operators.

The perturbations $u^{\prime}, \xi^{\prime}$, and $T^{\prime}$ correspond to axisymmetric perturbations. If all eigenvalues corresponding to these perturbations have negative real part, then the steady solution $u_{0}, \xi_{0}, T_{0}$ is a linearly stable solution of the axisymmetric equations (9) - (13). For this steady solution to have a corresponding linearly stable solution of the full three-dimensional model (2) - (4), we must also compute the eigenvalues corresponding to the non-axisymmetric perturbations. To do this, we linearize the perturbation equations corresponding to the 
three-dimensional model, and we assume that the eigenfunctions have the form

$$
\Phi(r, \varphi, \theta)=\hat{\Phi}_{m}(r, \theta) e^{i m \varphi}
$$

where $m=1,2, \ldots$ is the azimuthal wave number, which results in a generalized eigenvalue problem of the form (21) for each wave number $m$. In order to make the calculations of the non-axisymmetric eigenvalues tractable, we assume that the reflectional symmetry about the equator is not broken, and that there is no flow across the pole.

\section{$4 \quad$ Numerical methods}

\subsection{Discretization}

Because it is not possible to find analytic solutions for either the steady solution or the eigenvalue problem, the solutions are approximated numerically. Second order centered finite differencing is used to discretize the spatial derivatives. We approximate the value of the unknown functions at the locations of $N \times N$ uniformly spaced grid points in the interior of the domain. Sufficient accuracy is obtained from approximating the solutions on a uniform grid because the boundary layers in the steady solution are not severe (see below). The values of $u$ and $T$ on the outer boundary and on the equator, as well as those of $T$ at the pole, are not determined by the boundary conditions, and must also be considered as unknowns. This leads to discretized solution vectors of size $3 N^{2}+5 N$. Discretization of the steady equations for $u, \xi$, and $T$ leads to a system of nonlinear algebraic equations that can be solved by Newton iteration and Keller continuation as explained in Section 4.2, to find an approximation of the steady solution.

For the numerical approximation of the eigenvalues, the linearized perturbation equations are discretized, and thus the values of the steady solution are only needed at specific locations (the grid points) and the computed approximations are used. That is, the linearization is made about the approximate solution. Thus, upon discretization the partial differential eigenvalue problem becomes a generalized matrix eigenvalue problem.

\subsection{Solution techniques}

We are interested in computing the steady solution for a wide range of parameter values. To do this, we implement pseudo-arclength continuation with the Keller correction condition (see e.g. [7]), and use a Newton method to solve the resulting equations. If a solution is known for a particular set of parameter values, then this method can be used effectively to follow solutions as a parameter is varied, i.e. to find a solution curve (with respect to the parameter). 
Here, we know that for $\Delta T=0$, the trivial solution satisfies the equations for $u, \xi$ and $T$. Thus, for $\Delta T$ small, the trivial solution is a reasonable prediction of the solution, and Newton's method is used for the correction. In psuedo-arclength continuation, the parameter is considered as an unknown, and initial guesses of the solution are found by following the tangent, or a secant line approximation, to the solution curve. Increments are made approximately along the solution curve, and not by incrementing the parameter. The Keller condition ensures that the corrections to the initial guesses occur approximately perpendicularly to the tangent. This method is particularly useful because it is able to compute solutions along the solution curve even when there is a limit point on the curve, i.e. when the solution curve turns back on itself. In practice, the evaluation of the Jacobian is expensive, and therefore, in order to reduce the number of Jacobian evaluations, we use a quasi-Newton method in which the Jacobian is not updated on each iteration.

The generalized matrix eigenvalue problem that results from the discretization of (21) is solved in Matlab using the implicitly restarted Arnoldi method [17], which is a memoryefficient iterative method for finding a specified number of the largest eigenvalues. A generalized Cayley transformation [7] is made so that the Arnoldi iteration finds the eigenvalues of interest. In particular, the generalized Cayley transformation

$$
\mathbf{C}(\mathbf{L}, \mathbf{A})=\left(\mathbf{L}-\alpha_{1} \mathbf{A}\right)^{-1}\left(\mathbf{L}-\alpha_{2} \mathbf{A}\right)
$$

maps eigenvalues $\lambda$ of the generalized matrix eigenvalue problem $\lambda \mathbf{A} v=\mathbf{L} v$ to eigenvalues $\sigma$ of the transformed matrix $\mathbf{C}(\mathbf{L}, \mathbf{A})$, such that the eigenvalues $\lambda$ with $\operatorname{Real}(\lambda)>\left(\alpha_{1}+\alpha_{2}\right) / 2$ are mapped to the eigenvalues $\sigma$ with $|\sigma|>1$, where $\alpha_{1}$ and $\alpha_{2}$ are the real parameters of the Cayley transformation. The parameters of the transformation can be chosen to improve convergence properties. The matrix $\mathbf{C}(\mathbf{L}, \mathbf{A})$ does not have to be formed explicitly, because the Arnoldi iteration only requires matrix-vector products involving $\mathbf{C}(\mathbf{L}, \mathbf{A})$ [17]. Thus, the

full sparseness properties of $\mathbf{L}$ and $\mathbf{A}$ can be exploited, and computer memory requirements can be reduced.

\section{$5 \quad$ Existence and stability results}

The specific values of the parameters that are used in the computations are listed in Table 1. While these parameters remain fixed, we vary the gap width $R$ (and thus $r_{b} \equiv r_{a}+R$ ) differential heating $\Delta T$, and the rate of rotation $\Omega$, and compute the azimuthal (or zonal) fluid velocity $u$, the stream function $\xi$, and temperature deviation $T$.

For $\Delta T=0$, there is no movement of the fluid. However, for all $\Delta T>0$ fluid motion is induced. For small values of $\Delta T>0$ a single convection cell develops which we call a Hadley cell. An example of a Hadley cell solution for gap width $R=4$, and rotation rate 


\begin{tabular}{|c||c|c|}
\hline$r_{a}$ & 10 & $\mathrm{~cm}$ \\
\hline$\nu$ & $1.01 e^{-2}$ & $\mathrm{~cm}^{2} / \mathrm{sec}$ \\
\hline$\kappa$ & $1.41 e^{-3}$ & $\mathrm{~cm}^{2} / \mathrm{sec}$ \\
\hline$\alpha$ & $2.06 e^{-4}$ & $1 /{ }^{\circ} \mathrm{C}$ \\
\hline$\rho_{0}$ & 0.998 & $\mathrm{gm} \mathrm{cm}{ }^{3}$ \\
\hline$T_{0}$ & 20.0 & ${ }^{\circ} \mathrm{C}$ \\
\hline$g$ & 980 & $\mathrm{gm} / \mathrm{cm}^{3}$ \\
\hline
\end{tabular}

Table 1: The parameters of the spherical shell and fluid used in the computations. See Section 2 for definitions of the symbols.

$\Omega=0.03$ is plotted in Figure 4. In the figure, the stream function $\xi=\xi(r, \theta)$, the azimuthal (zonal) velocity $u=u(r, \theta)$, and the temperature deviation from the temperature on the inner boundary $T=T(r, \theta)$, are plotted on the domain $r_{a} / R \leq r \leq r_{b} / R, 0 \leq \theta \leq \pi / 2$. The radii of the inner and outer boundaries have been mapped to 1 and 2 respectively so that the flow in the interior of the shell is more readily visualized. The figure represents a cross-section of the flow at an arbitrary value of the azimuthal variable $\varphi$. The solution corresponding to the full equations (2)-(4) is obtained by rotating about the polar axis and reflecting across the equator. The ' + ' and ' - ' indicate the contours corresponding to positive and negative values of the functions, respectively, while contours that intersect the inner boundary necessarily correspond to zeros of the functions. The polar velocity $v$ and the radial velocity $w$ can be inferred from the stream function $\xi$ using (17). In particular, the flow tends to follow contours of $\xi$, where the arrows in the figure indicate the direction of the flow along the contours. For this figure and all others that follow, we have taken $N=40$.

In the flow depicted in Figure 4 the fluid rises at the equator and falls at the pole. The azimuthal velocity $u$ attains a maximum in the mid-latitudes $(\theta \sim \pi / 6)$ near the outer boundary. This is remarkably similar to the azimuthally averaged azimuthal velocities observed in the Earth's atmosphere, except that in the atmosphere the jet stream occurs at a somewhat lower latitude (see, e.g., [26]).

The eigenvalues with the 10 largest real parts associated with the axisymmetric eigenfunctions, as well as those associated with the eigenfunctions for each wave number between 1 and 8 are approximated using the techniques described in Section 3. It is found that all eigenvalues that are computed have negative real part. For the larger values of the wave number $m$, the eigenvalues become more negative as the wave number is increased. Thus we expect that all eigenvalues associated with all wave numbers have negative real parts, and we conclude that the circulation pattern in Figure 4 is a linearly stable solution of the full three-dimensional equations.

If the heating parameter $\Delta T$ is increased further while keeping the gap width $R$ fixed, 

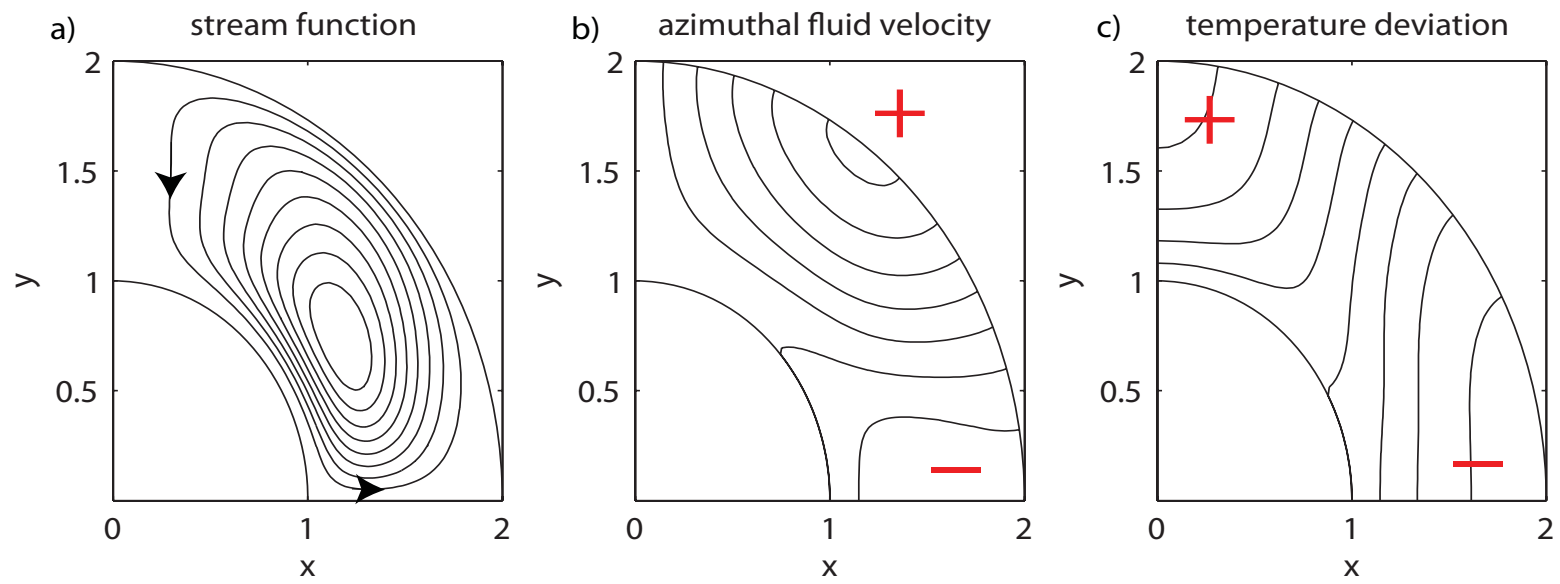

Figure 4: An example of a single-cell circulation pattern observed for heating parameter $\Delta T=0.004$, gap width $R=4$, and rotation rate $\Omega=0.03$. (a) The stream function $\xi$; flow tends to follow contours, (b) the azimuthal (or zonal) velocity $u$, and (c) the temperature deviation $T$ from the temperature prescribed on the lower boundary. The inner and outer boundaries have been mapped to $r=1$ and $r=2$, respectively.

a transition is observed. First, the flow passes through an intermediate stage shown in Figure 5, then as $\Delta T$ is increased further, a three-cell pattern develops (see Figure 6). The three-cell pattern resembles the zonally (azimuthally) averaged circulation pattern observed in the Earth's atmosphere, with a strong cell close to the equator (the Hadley cell), a weaker counter-rotating cell in the midlatitude (sometimes called the Ferrel cell), and finally an even weaker cell near the pole with the same direction of rotation as the Hadley cell [26]. It is found that these solutions are also linearly stable to both axisymmetric and non-axisymmetric perturbations.

It is of particular interest that, although there is clearly a transition in the flow pattern as $\Delta T$ is increased, there is no point at which the solution is neutrally stable, i.e. no eigenvalue crosses the imaginary axis. This is not entirely unexpected. Other studies in which flow transitions in systems with a lack of symmetry were investigated have revealed such behaviour, for example see $[14,27]$. Such a transition may be induced by a broken pitchfork bifurcation or a perturbed hysteresis bifurcation (see below). Therefore, we search for such a mechanism.

Although our full system has $S O(2) \times \mathbb{Z}_{2}$ symmetry, we are looking for solutions that preserve this symmetry, so we have used the symmetry to simplify the equations for $u, \xi$ and $T$. The simplified equations possess none of these symmetries. The symmetries of a system determine the types of bifurcation that are likely to occur, i.e. which types are generic for the system. For equations with no symmetry, saddle-node bifurcations are generic and are expected to be observed if a real eigenvalue crosses through zero as a single parameter is varied. It is also possible for a Hopf bifurcation to occur, if a complex eigenvalue crosses the 

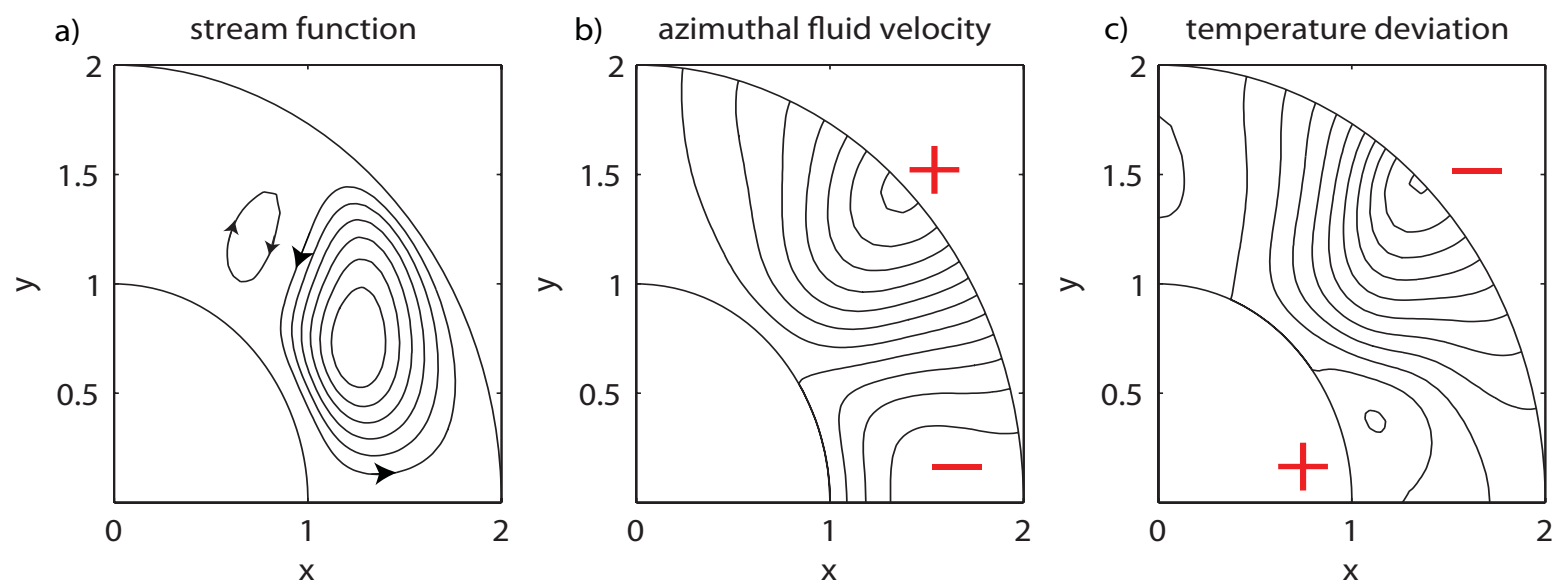

Figure 5: An intermediate stage circulation pattern observed for heating parameter $\Delta T=0.0133$, gap width $R=4$, and $\Omega=0.03$. (a) The stream function $\xi$; flow tends to follow contours, (b) the azimuthal (or zonal) velocity $u$, and (c) the temperature deviation $T$ from the temperature prescribed on the lower boundary. The inner and outer boundaries have been mapped to $r=1$ and $r=2$, respectively.

imaginary axis with a nonzero imaginary part. However, Hopf bifurcations involve transitions from steady solutions to periodic orbits, and since we consider only steady solutions in this paper, we do not seek Hopf bifurcations.

The presence of a saddle-node bifurcation in itself can not explain the observed transition, which occurs without a zero eigenvalue. However, such a transition may occur near an organizing center or bifurcation of codimension 2. This implies that it will be necessary to vary a second parameter to find it. The types of codimension 2 bifurcation points that could occur generically in a system like ours are the broken pitchfork bifurcation $[9,14,27]$ and the hysteresis (or cusp) bifurcation as described in the introduction and in $[9,15]$.

In order to clarify the origin of this transition, it is useful to explore the solutions at larger gap width and rate of rotation. If the gap width is increased to $R=10$ and the rotation rate to $\Omega=0.05$, then, when the heating $\Delta T$ is small enough, the linearly stable one-cell pattern is maintained, with a slight distortion of the stream function near the outer boundary. An example is plotted in Figure 7. We see that as the heating parameter $\Delta T$ is increased, there is a transition to a two-cell pattern (Figure 8), and again this transition occurs without an eigenvalue corresponding to an axisymmetric eigenfuction crossing the imaginary axis.

It is observed that as the gap width $R$ and the rotation rate $\Omega$ are increased, the steady solutions become less stable to non-axisymmetric perturbations. As a result, although this two-cell pattern is stable to axisymmetric perturbations, it is linearly unstable to nonaxisymmetric perturbations. The loss of stability to the non-axisymmetric perturbations occurs as a Hopf bifurcation, and thus the bifurcating solution is expected to be a rotating 

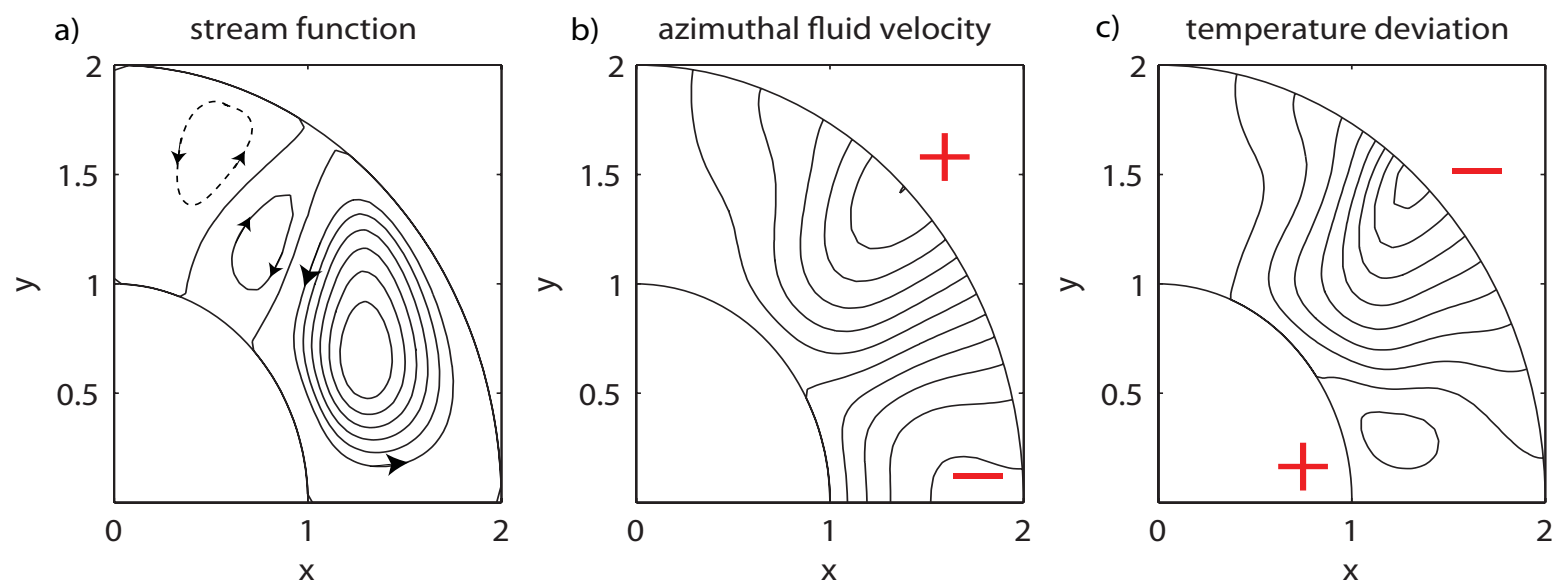

Figure 6: An example of a three-cell circulation pattern observed for heating parameter $\Delta T=0.025$, gap width $R=4$, and rotation rate $\Omega=0.03$. (a) The stream function $\xi$; flow tends to follow contours, (b) the azimuthal (or zonal) velocity $u$, and (c) the temperature deviation $T$ from the temperature prescribed on the lower boundary. The inner and outer boundaries have been mapped to $r=1$ and $r=2$, respectively. The dashed line represents an approximate contour corresponding to a value of the stream function that is $-1 / 3$ of the increment between the solid contour values.

wave with azimuthal wave number $m$. This implies that this two-cell solution will not be physically observable directly. However, because the loss of stability occurs near the one-cell to two-cell transition, it is possible that the rotating wave will inherit the $\theta$ and $r$ dependence of the two-cell solution, i.e. an azimuthal average of the rotating wave will have a two-cell structure. We do not show this here, as it is secondary to our purpose for presenting this example. Regardless of the stability with respect to non-axisymmetric perturbations, the two-cell pattern is a linearly stable solution of the axisymmetric equations, and forms in qualitatively the same manner as the transition that is observed at at lower $R$ and $\Omega$ (see below for further discussion). Thus, an investigation of the transition to this flow, in the axisymmetric equations, will provide a mechanism for the transition. Below we consider only solutions and stability with respect to the axisymmetric equations.

As mentioned above, the transition at $R=10$ and $\Omega=0.05$ occurs without an eigenvalue corresponding to an axisymmetric eigenfuction crossing the imaginary axis. Because the eigenvalue with largest real part is real, this implies that there is no parameter value at which there is a zero eigenvalue. In Figure 9, we plot the real value of the (axisymmetric) eigenvalue with largest real part as a function of the heating parameter $\Delta T$, and we plot a "bifurcation diagram" that indicates how the solutions change as $\Delta T$ changes. The vertical axis is the parameter $s$, which is the distance travelled along the solution curve. This measure is chosen because it highlights the main features most effectively. In the figure it can be seen that as the heating parameter $\Delta T$ increases, the value of the eigenvalue increases 

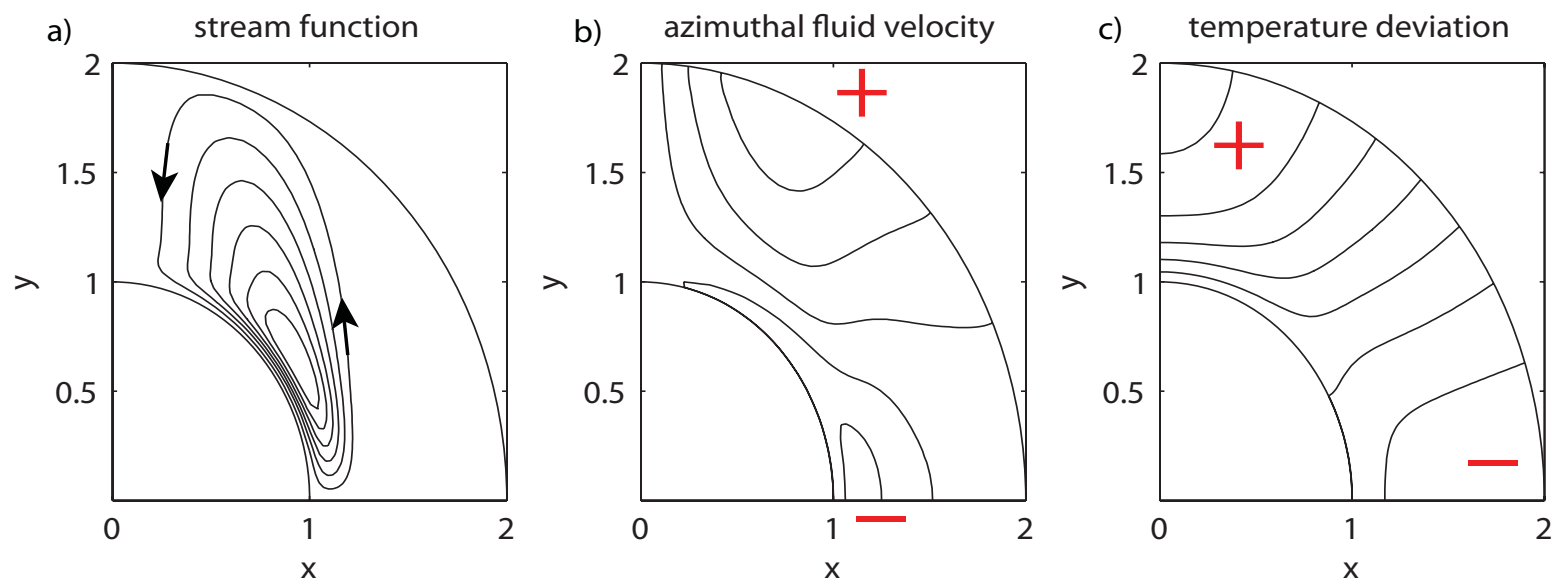

Figure 7: An example of a single-cell circulation pattern observed for heating parameter $\Delta T=0.002$, gap width $R=10$, and rotation rate $\Omega=0.05$. (a) The stream function $\xi$; flow tends to follow contours, (b) the azimuthal (or zonal) velocity $u$, and (c) the temperature deviation $T$ from the temperature prescribed on the lower boundary. The inner and outer boundaries have been mapped to $r=1$ and $r=2$, respectively.

until it reaches a maximum that is negative, at which point it begins to decrease without ever reaching zero. Heuristically, it is observed that the development of the two-cell pattern begins to occur near this maximum.

If the gap width is increased to $R=35$, then for small values of $\Delta T$ a linearly stable one-cell pattern is again observed. See Figure 10. A gap width of $R=35$ corresponds to an aspect ratio $\eta=R / r_{a}=3.5$, i.e. the gap is three and a half times larger than the radius of the inner sphere. Although we are not necessarily interested in flows at such a large aspect ratio, they will help to explain the transition that is observed for smaller gap width.

As we increase the differential heating $\Delta T$, again we see a transition from the one-cell to a two-cell pattern, shown in Figure 11. However, in this case, a real eigenvalue does cross the imaginary axis. In Figure 12, we plot both the real part of the eigenvalue with largest real part as a function of $\Delta T$, and the corresponding bifurcation diagram. The crossing of the imaginary axis by a real eigenvalue corresponds to a saddle-node bifurcation, also referred to as a limit point or fold. As the solution curve is followed past the bifurcation point, the solution becomes linearly unstable, and the value of $\Delta T$ begins to decrease. For a short interval, the real eigenvalue increases until it reaches a maximum, after which it begins to decrease. Subsequently, it again crosses the imaginary axis, and a second saddle-node bifurcation is observed. As we follow the curve past this point, the solution returns to being linearly stable, and $\Delta T$ once again begins to increase. This pair of saddle-node bifurcations results in an 'S' shaped bifurcation diagram. As we follow the solution curve, from the lower part of the 'S' to the upper part of the 'S', the real part of the eigenvalue with largest real part traces out the loop seen in Figure 12. 

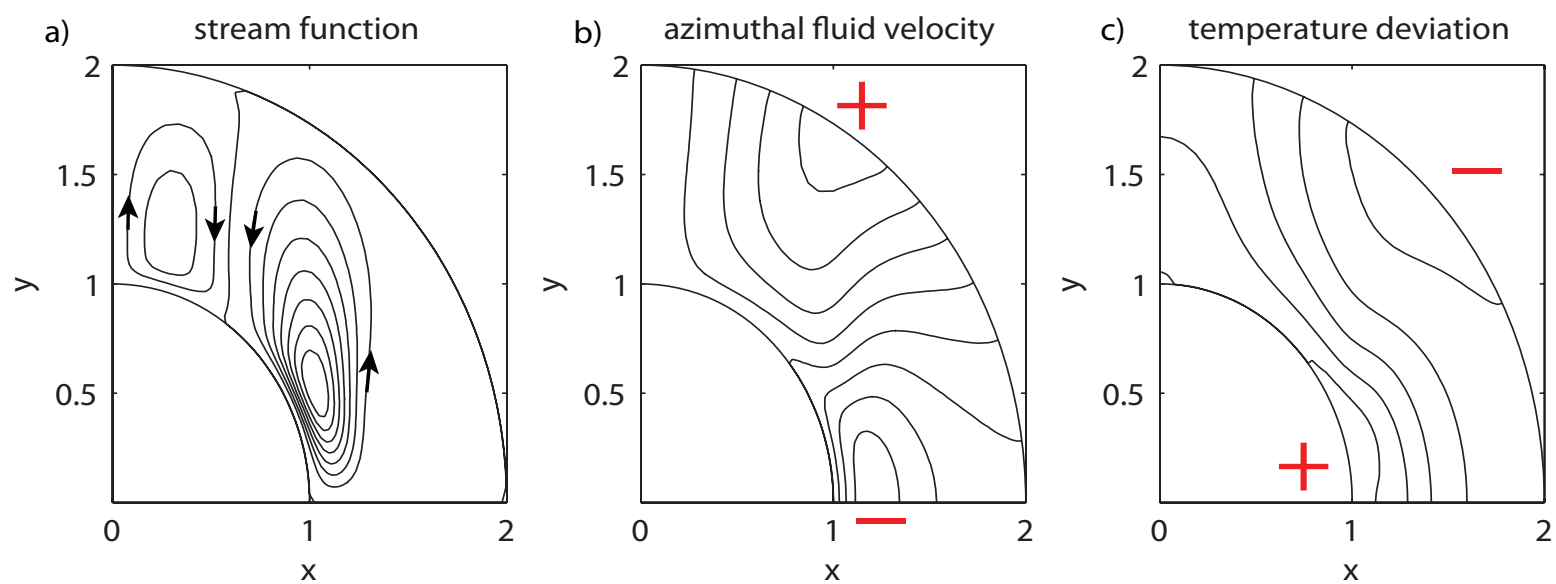

Figure 8: An example of a two-cell circulation pattern observed for heating parameter $\Delta T=0.029$, gap width $R=10$, and rotation rate $\Omega=0.05$. (a) The stream function $\xi$; flow tends to follow contours, (b) the azimuthal (or zonal) velocity $u$, and (c) the temperature deviation $T$ from the temperature prescribed on the lower boundary. The inner and outer boundaries have been mapped to $r=1$ and $r=2$, respectively.

This pair of saddle-node bifurcations is a classical mechanism for hysteresis. In a physical observation of such a system, the solution on the bottom branch abruptly disappears as $\Delta T$ is increased past the first limit point, and the system equilibrates to the stable solution on the upper branch. If $\Delta T$ is decreased from this point, the solution on the upper branch remains stable until it also abruptly disappears as $\Delta T$ is decreased past the second limit point. Thus, the two transitions between the two solutions do not occur at the same value of $\Delta T$ when $\Delta T$ is increasing or decreasing, and there is an interval between the transitions in which both solutions are stable (bistability).

\section{Hysteresis bifurcation}

The behaviour observed in Figures 9 and 12 is indicative of a hysteresis (or cusp) bifurcation point. The typical behaviour of a nonlinear system near a hysteresis point is as shown in Figure 3. It can be expected that at some critical value of the gap width $R=R_{c}$, a one-dimensional bifurcation diagram in $\Delta T$ will have a single vertical tangent, with slopes on either side being positive. For values of $R<R_{c}$, we expect no bifurcations as $\Delta T$ is increased (as in Figure 9), while for $R>R_{c}$ we expect a pair of saddle-node bifurcations (as in Figure 12). This behaviour can be seen in Figure 3, where these different one-dimensional bifurcation diagrams in $\Delta T$ may be obtained as slices through $M$, taken with different values of constant $R$. Mathematically, a hysteresis or cusp bifurcation point is determined by the following three conditions [15]:

1. There exists a steady solution. 

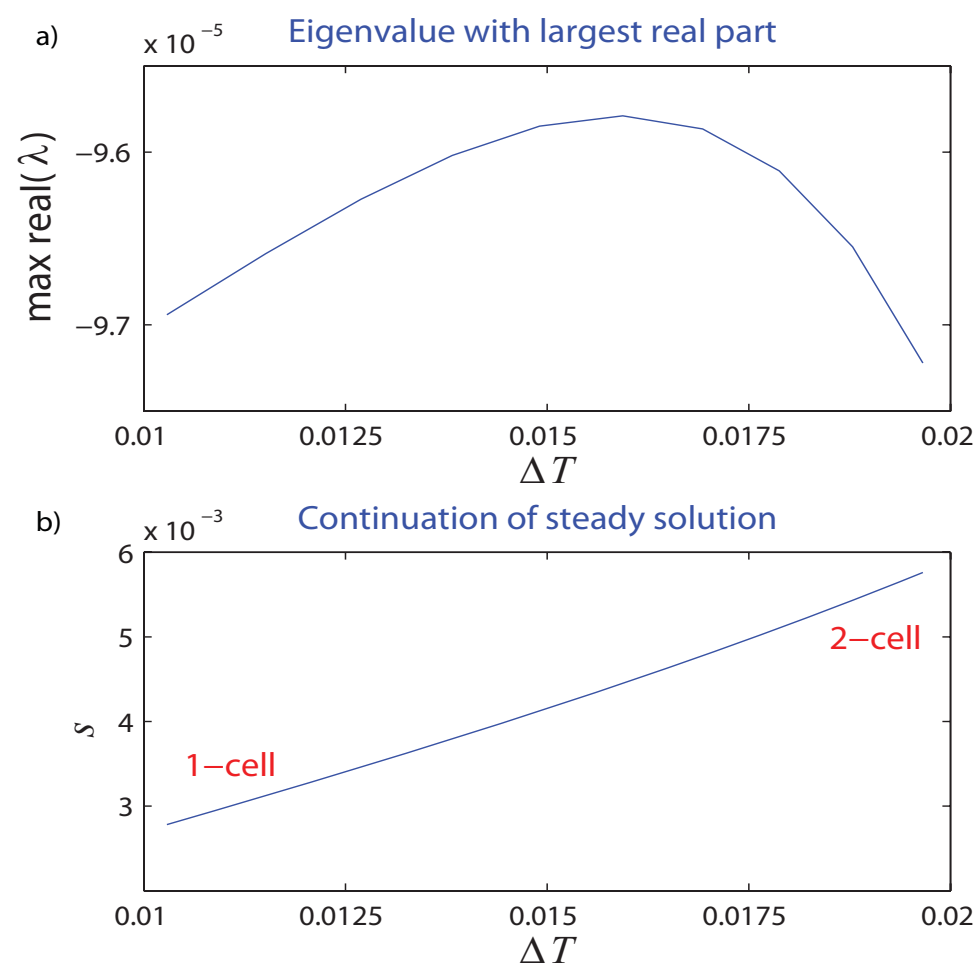

Figure 9: Results for $R=10, \Omega=0.05$. (a) Real part of eigenvalue with largest real part versus $\Delta T$, and (b) Bifurcation diagram in $\Delta T$; vertical axis represents distance along solution curve.

2. There is a simple zero eigenvalue of the linearization of the equations about the steady solution.

3. The coefficient of the second order term of the normal form on the centre manifold vanishes.

Therefore, in order to demonstrate that the observed behaviour is indeed generated by a hysteresis bifurcation, it is necessary to show that each of the above three conditions is satisfied. Although it may seem that this would be a daunting task, in fact these conditions can be verified by an explicit calculation that can be performed numerically as follows, see $[7,15]$. To elucidate these conditions and the means that we use to compute the hysteresis point, we write the equations for $\xi, u$, and $T$ in the abstract form:

$$
\dot{U}=L U+N(U, U)
$$

where

$$
U=\left(\begin{array}{c}
\xi \\
u \\
T
\end{array}\right)
$$



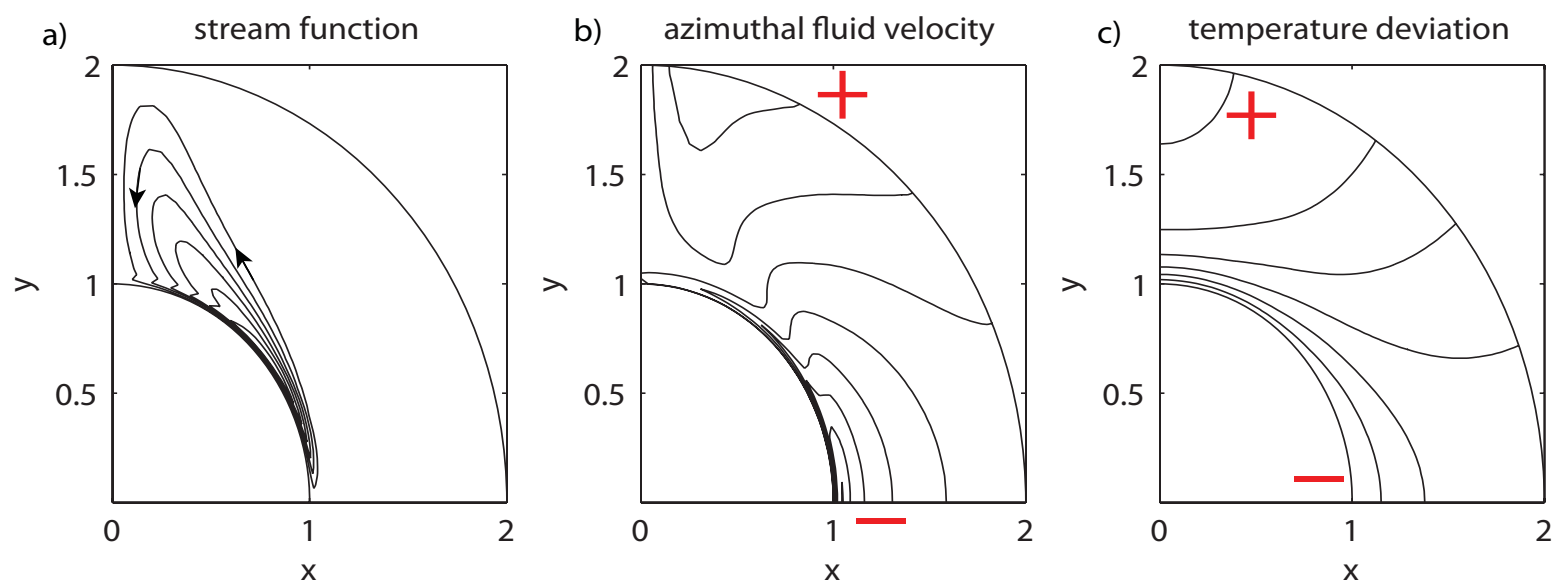

Figure 10: An example of a single-cell circulation pattern observed for heating parameter $\Delta T=0.001$, gap width $R=35$, and rotation rate $\Omega=0.05$. (a) The stream function $\xi$; flow tends to follow contours, (b) the azimuthal (or zonal) velocity $u$, and (c) the temperature deviation $T$ from the temperature prescribed on the lower boundary. The inner and outer boundaries have been mapped to $r=1$ and $r=2$, respectively.

is the dependent variable, $L$ is the linear operator such that $L U$ is the linear part of the equations, $N(U, U)$ is the nonlinear part of the equations, and the dot represents differentiation with respect to time. Note that the nonlinear part $N$ is only quadratic (from the Navier-Stokes equation).

Assume that for some critical values of the parameters $(\Delta T, R)=\left(\Delta T_{c}, R_{c}\right)$, there is a steady solution $U_{0}$ of (23), i.e., $U_{0}$ satisfies

$$
L U_{0}+N\left(U_{0}, U_{0}\right)=0
$$

Assume also that at $(\Delta T, R)=\left(\Delta T_{c}, R_{c}\right)$ the linearization $L_{0}$ about the steady solution $U_{0}$ has a simple zero eigenvalue $\lambda_{0}=0$ while all other eigenvalues have negative real part, where $L_{0}$ is given by

$$
L_{0} V=L V+N\left(V, U_{0}\right)+N\left(U_{0}, V\right)
$$

That is, we have

$$
L_{0} \Psi=0
$$

where $\Psi$ is the eigenfunction corresponding to the zero eigenvalue.

Under certain conditions on $L_{0}$, the dependent variable $U$ can be written in the form

$$
U=w \Psi+\Phi
$$

where $w \in \mathbb{R}$ and thus $w \Psi \in \operatorname{span}\{\Psi\}$, and $\Phi \in E_{s}$. Here $E_{s}$ is called the stable subspace, and is the space spanned by all eigenfunctions corresponding to eigenvalues with negative real part. 

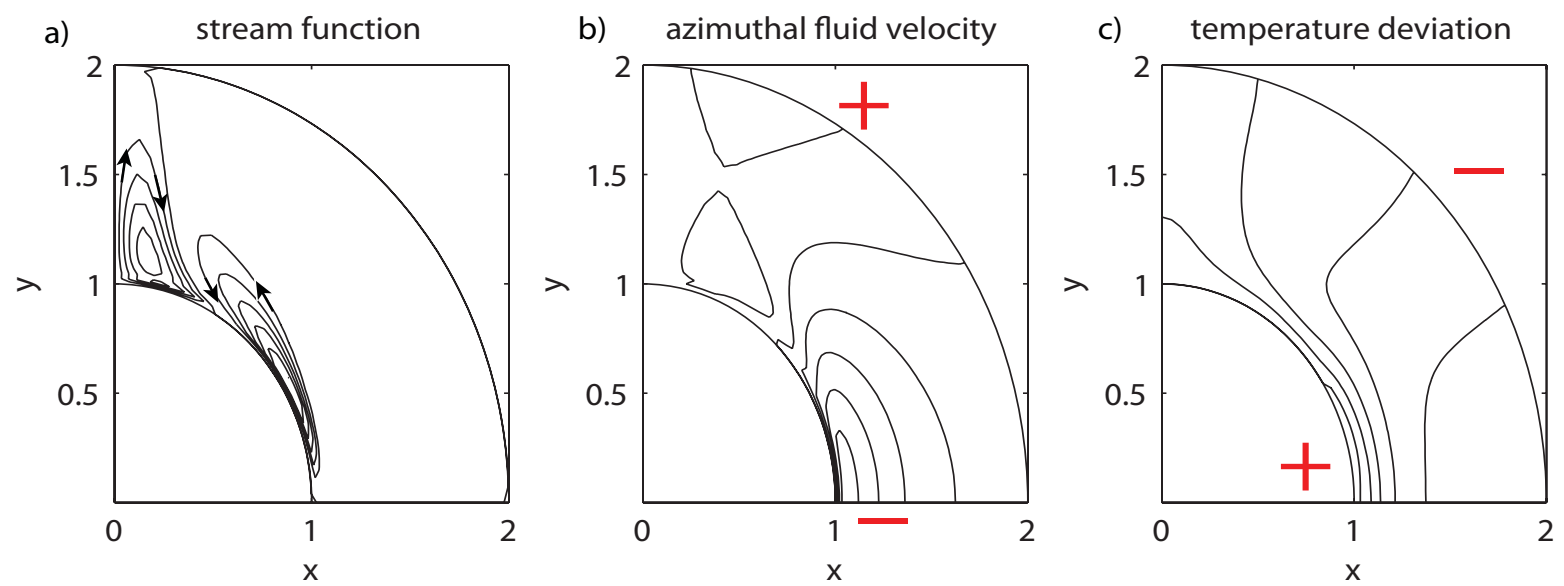

Figure 11: An example of a two-cell circulation pattern observed for heating parameter $\Delta T=0.019$, gap width $R=35$, and rotation rate $\Omega=0.05$. (a) The stream function $\xi$; flow tends to follow contours, (b) the azimuthal (or zonal) velocity $u$, and (c) the temperature deviation $T$ from the temperature prescribed on the lower boundary. The inner and outer boundaries have been mapped to $r=1$ and $r=2$, respectively.

If we write $U$ as in (27) then under certain technical conditions, a centre manifold and normal form reduction can be performed on (23) to obtain the equation on the centre manifold in normal form

$$
\dot{w}=\beta_{1}+\beta_{2} w+a w^{2}+c w^{3}+O\left(w^{4}\right),
$$

where $a$ and $c$ are coefficients of the normal form, and $\beta_{1}$ and $\beta_{2}$ are unfolding parameters that are in general functions of the parameters $\Delta T$ and $R$. It can be shown that if $c \neq 0$, then neglecting the terms of $O\left(w^{4}\right)$ do not change the qualitative features of the solutions.

The centre manifold and normal form theories state that for $(\Delta T, R)$ near $\left(\Delta T_{c}, R_{c}\right)$ and when the solutions are in some sense small, then the dynamics of (23) can be deduced from (28). In particular, solutions of (28) are in one-to-one correspondence with those of (23).

Formulas for the coefficients of the normal form equation can be derived by performing a centre manifold and normal form reduction in the general case $[7,15]$. In particular, the coefficient of the second order term is given by

$$
a=1 / 2\left\langle\Psi^{*}, N(\Psi, \Psi)\right\rangle,
$$

where $\Psi$ is the eigenfunction corresponding to $\lambda_{0}, \Psi^{*}$ is the corresponding adjoint eigenfunction corresponding to $\lambda_{0}$, and

$$
\langle U, V\rangle=\iint U \cdot V d \mathbf{r}
$$

is the inner product on the domain.

For $a=0$, a hysteresis bifurcation occurs when $\beta_{1}=\beta_{2}=0$. For $\beta_{2} / c>0$, there is a single solution to (28) for all $\beta_{1}$. For $\beta_{2} / c<0$, there is a region in the two-dimensional 

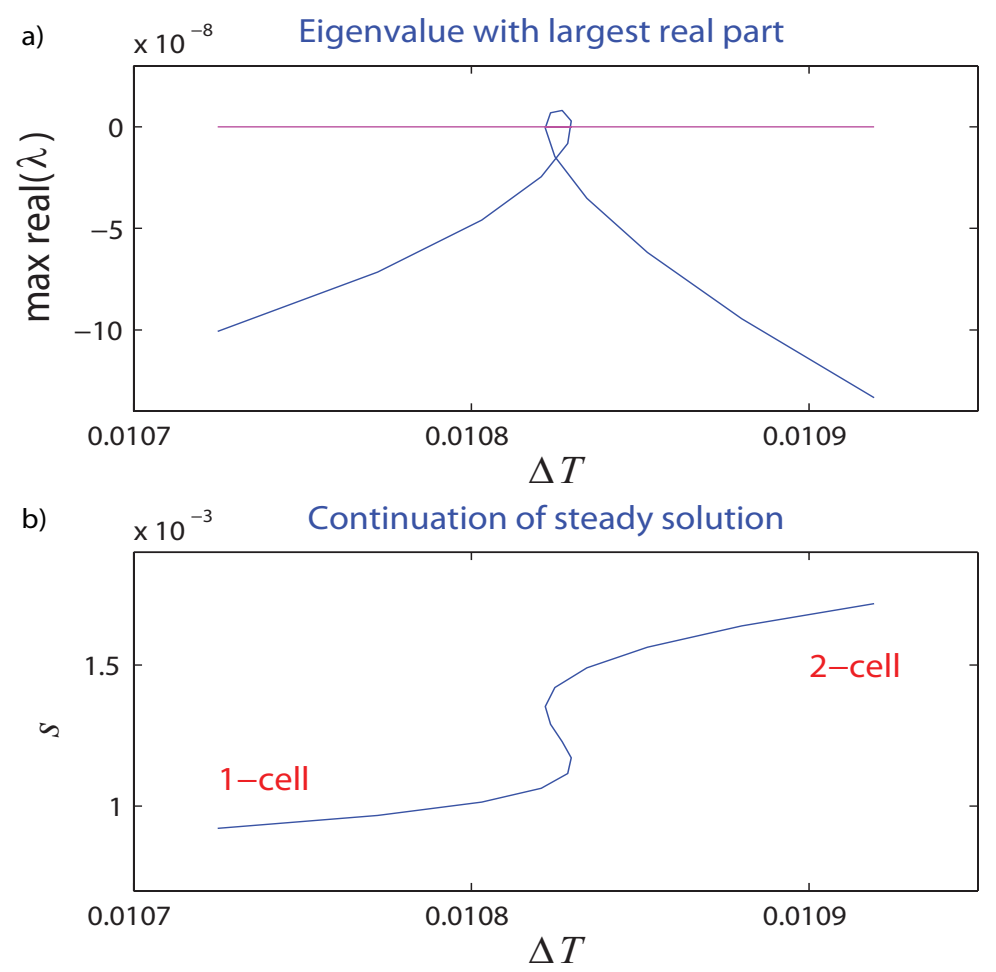

Figure 12: Results for $R=35$. (a) Real part of eigenvalue with largest real part versus $\Delta T$, and (b) Bifurcation diagram in $\Delta T$; vertical axis represents distance along solution curve.

parameter space $\left(\beta_{1}, \beta_{2}\right)$ in which there are three solutions. The borders of this region are given asymptotically by the two curves

$$
\beta_{1}= \pm \frac{2}{3} \sqrt{\frac{-\beta_{2}}{c}} \beta_{2}
$$

As $\beta_{2}$ approaches zero, these two curves approach each other and meet in a cusp at $\beta_{1}=$ $\beta_{2}=0$. This is the origin of the name "cusp bifurcation".

In order to show that a hysteresis or cusp bifurcation occurs in the model, we need to show that the three aforementioned conditions are satisfied. That is, we must find parameter values $(\Delta T, R)=\left(\Delta T_{c}, R_{c}\right)$, such that the following three equations are satisfied

$$
\begin{aligned}
L U_{0}+N\left(U_{0}, U_{0}\right) & =0, \\
L_{0} V & =0, \quad\langle V, V\rangle=1, \\
a=1 / 2\left\langle\Psi^{*}, N(\Psi, \Psi)\right\rangle & =0,
\end{aligned}
$$

where $L_{0}$ is given by $(25)$.

These equations have the unfortunate property that for some values of $\Delta T \neq \Delta T_{c}$, $R \neq R_{c}, L_{0}$ will not be singular, and thus (33) will not have a solution for any $V$. Therefore, 
it will be convenient to use the following defining system [7]

$$
\begin{aligned}
L U_{0}+N\left(U_{0}, U_{0}\right) & =0 \\
g & =0 \\
g^{\prime} & =0
\end{aligned}
$$

where $g$ and $g^{\prime}$ are scalars given by

$$
\begin{gathered}
L_{0} V+g B=0, \quad\langle C, V\rangle=1, \\
L_{0} V^{\prime}+g^{\prime} B=-N(V, V), \quad\left\langle C, V^{\prime}\right\rangle=0,
\end{gathered}
$$

and where $B$ is not in the range of $L_{0}$, and $C$ is not in the range of the adjoint operator $L_{0}^{*}$, which is defined by

$$
\left\langle L_{0} U, V\right\rangle=\left\langle U, L_{0}^{*} V\right\rangle
$$

for all $U$ and $V$.

Each of the three equations $(35)$ - (37) corresponds to one of the hysteresis point defining conditions. Specifically, $U_{0}$ is a steady solution of (23) when the first equation (35) is satisfied. If we set $g=0$ in (38) and there is a solution, then $L_{0}$ has a zero eigenvalue with corresponding eigenfunction $V$. Thus, the second hysteresis condition is satisfied when the second equation (36) is satisfied. In this case, when $L_{0}$ is not singular then there will still be a solution of (38), namely one for which $g \neq 0$. This is assured by choosing $B$ not in the range of $L_{0}$. If we set $g^{\prime}=0$ in (39), and there is a solution, then we have that $N(V, V)$ must be in the range of $L_{0}$. If we also have $g=0$, then from above we have that $V$ is the eigenfunction of $L_{0}$ with zero eigenvalue, i.e. $V=\Psi$. Thus, if $N(V, V)$ is in the range of $L_{0}$ then, by the "Fredholm alternative" property of the adjoint, $N(V, V)$ must be orthogonal to the eigenfunction of $L_{0}^{*}$ corresponding to the zero eigenvalue, i.e. we must have $\left\langle\Psi^{*}, N(\Psi, \Psi)\right\rangle=0$, and thus the second-order coefficient of the normal form vanishes. When $L_{0}$ is nonsingular, (39) has a solution regardless of $g^{\prime}$. However, there is also the possibility that close to the cusp there are parameter values such that $L_{0}$ is singular, while $N(V, V)$ is not in the range of $L_{0}$; this occurs at the saddle-node bifurcations. Thus, there will be no solution of (39) for $g^{\prime}=0$. However, because $B$ is not in the range of $L_{0}$, we are assured a solution with $g^{\prime} \neq 0$.

In order to make the equations linear in $V$ and $V^{\prime}$, we can choose the normalization conditions as shown. Solutions are assured when $C$ is chosen to be not in the range of the adjoint operator $L_{0}^{*}$. In practice, because the kernel of $L_{0}$ is only one dimensional, it is easy to choose $B$ and $C$ with the required properties. 
It can be proved that when the system (35) - (37) has a solution, then not only is the nondegeneracy condition for a cusp bifurcation satisfied, but also the transversality condition [7]. Thus, we are assured that we have found a cusp bifurcation.

Upon discretization of (35) - (37) and (38) - (39) on an $N \times N$ grid, we obtain a system of nonlinear algebraic equations. For various values of $N$, we find that there are critical parameter values $(\Delta T, R)=\left(\Delta T_{c}, R_{c}\right)$ such that there is a $U_{0}, g$ and $g^{\prime}$ that satisfy the discretized system. Results are listed in Table 6 . It can be seen that the difference between the results for $N=40$ and $N=80$ is approximately twice the difference between results for $N=80$ and $N=160$. Thus, the results provide evidence that the numerical results are convergent with order approximately $O(h)$. We can conclude that a hysteresis bifurcation does in fact exist in the model with cusp point $\left(\Delta T_{c}, R_{c}\right) \approx(0.013,38)$.

\begin{tabular}{|c||c|c|}
\hline$N$ & $\Delta T$ & $R$ \\
\hline 40 & 0.01080 & 34.63 \\
\hline 80 & 0.01255 & 37.61 \\
\hline 160 & 0.01347 & 38.07 \\
\hline
\end{tabular}

Table 2: Critical parameter values at which the hysteresis bifurcation occurs, for various values of $N$. The results provide evidence of $O(h)$ convergence.

This provides strong evidence that the transition from the one-cell to the two-cell pattern, that is observed at gap width $R=10$ as the differential heating $\Delta T$ is increased, is explained by this cusp bifurcation. We also postulate that the transition from the one-cell to the threecell pattern, that is observed at gap width $R=4$ as the differential heating $\Delta T$ is increased, is not only the same mechanism, but is associated with the same bifurcation. This is evident if we consider the transition from the one-cell pattern for a sequence of gap widths $R$ and rotation rates $\Omega$ that decrease from $R=10, \Omega=0.05$ to $R=4, \Omega=0.03$. For all transitions in this sequence, regardless of whether the transition results in a two-cell or three-cell pattern, the eigenvalue with largest real part behaves in the same manner (as shown in Figure 9) with only small quantitative changes. Furthermore, in all cases, the stream function component of the eigenfunctions corresponding to the eigenvalue with largest real part has a two-cell structure. The azimuthal velocity component of the eigenfunctions also shows little variation. In all cases, this component is similar to the azimuthal velocity itself before transition, which explains the lack of a qualitative change in the azimuthal velocity in the transitions. None of these results provide any evidence that another bifurcation is taking place.

Furthermore, there is a smooth variation of the qualitative behaviour as $R$ is varied. For large $R$, the transition from one-cell to two-cell begins with a flattening of the stream function $\xi$ near the pole, corresponding to a decrease in fluid velocity in this region, and is followed by 
the formation of the counter-rotating cell near the pole. The new cell is first observed as a small cell adjacent to the pole, and grows as the differential heating $\Delta T$ is increased. Changes in the rotation rate $\Omega$ affect the stability of the solution to non-axisymmetric perturbations, but do not affect the qualitative features of the transition, and therefore, here, we refer only to changes in gap width $R$. If $R$ is decreased, the flattening becomes more pronounced before the second cell is observed. When the cell is observed, it grows more quickly with $\Delta T$ than when the gap width is larger. If the differential heating is increased sufficiently, a transition from the two-cell pattern to a three-cell pattern is observed. For yet smaller gap width, (e.g. $R=4$ ), again the transition begins with a flattening. However, in this case, the second cell does not first appear adjacent to the pole, but peeks out a small distance from the pole. This transitional stage, shown in Figure 5, is not in essence a two-cell pattern, because between the new cell and the pole there is a very weak (almost quiescent) region in which the fluid rotates in the same sense as the large cell near the equator.

The development of the two-cell pattern is easily explained in terms of the lowest order dynamics of the cusp bifurcation. To lowest order, the solution $U$ to the perturbation equations will be given by $w \Psi$, where $w \in \mathbb{R}$, and $\Psi$ is the eigenfunction corresponding to the eigenvalue with largest real part, see (27). Thus, to first order, the solution to the axisymmetric equations will be $w \Psi$ plus the solution about which we have linearized, i.e., the one-cell solution. Thus, because the stream function component of the eigenfunction $\Psi$ has a two-cell structure, we expect that, to lowest order, the solution will also develop a two-cell structure.

However, if we wish to compute the solution to higher order, it would be necessary to explicitly compute some coefficients of the centre manifold function. These coefficients will depend on the nonlinear part of the equations, and thus will involve the nonlinear interaction of the different components of the eigenfunction. In particular, we expect that if the higherorder terms are sufficiently large, then this term may induce a three-cell pattern. We expect that these effects will become more important as we increase the differential heating $\Delta T$ away from the initial transition. Also, due to the factor of $1 / R$ that multiplies the nonlinear part of the equations, we expect that these effects will become more significant as $R$ is decreased. These are both consistent with the observed results.

We have already pointed out that the variation of the rotation rate $\Omega$ has relatively smaller qualitative effect on the solutions of the axisymmetric equations. Indeed, a cusp is observed if the rotation is held fixed at $\Omega=0.03$, and only the gap width $R$ is increased. We choose not to present this example because, in this case, an additional saddle-node bifurcation occurs at values of the differential heating slightly larger than where the cusp is observed. As the rotation rate is increased, this bifurcation moves further away from the 
cusp, and thus the example for $\Omega=0.05$ more clearly indicates the origin of the observed transitions.

\section{Discussion and Conclusions}

This work has shown that a Boussinesq fluid in a rotating spherical shell, differentially heated on its inner surface, can exhibit a variety of stable rotationally symmetric flow patterns. Distinctive features of these flow patterns include a Hadley cell with a flow pattern much like the Hadley cells of the Earth, and a high azimuthal velocity jet stream located at high altitudes and mid-latitudes, much like the jet stream in each hemisphere of the Earth. For small values of the differential heating parameter $\Delta T>0$, the Hadley cell exists and extends from equator to pole. For larger values of $\Delta T$, first one then two or more additional convection cells may form between the Hadley cell and the pole in the mid and polar latitudes. The first cell to form after the Hadley cell exhibits counter-rotating flow (westerly winds near the inner surface) and the third cell has Hadley-like rotation (easterly winds near the polar surface). The observed transitions are distinctly related to the spherical geometry of the system. This is evident because in all models of differentially heated fluids in domains with cylindrical geometry, such transition that do not break the rotational symmetry only occur at very high differential heating.

The features of the transitions are not affected by moderate changes in the rate of rotation $\Omega$, although the solutions become less stable to non-axisymmetric perturbations as $\Omega$ is increased. However, changes in the gap width $R$ can induce significant changes. Mathematical analysis of the axisymmetric model demonstrates that it possesses a codimension two hysteresis (or cusp) bifurcation for a critical choice of the parameters $(R, \Delta T)=\left(R_{c}, \Delta T_{c}\right)$. In a neighborhood of this hysteresis point, for larger $R$, there exists a region of bistability in which two different states of the system are both linearly stable solutions of the axisymmetric equations. These two stable states are separated by a third unstable state. For such $R$ (fixed), there exists an interval of values of $\Delta T$ exhibiting a hysteresis loop as illustrated in Figure 3. At each end of this interval, a small change in $\Delta T$ can cause a transition in the state of the system, to a qualitatively different flow pattern, e.g. one with a different number of convection cells.

In the model, changes in $\Delta T$ have a direct influence on changes in the Hadley cell, but changes in the Hadley cell have no influence on the temperature difference parameter $\Delta T$. This is not the case in a real planetary system, where convection cells are known to act as "thermal conveyor belts". For example, a Hadley cell transports heat away from the equatorial regions to higher latitudes. In the case of small $\Delta T$ in which a large Hadley cell extends from equator to pole, this conveyor belt would have the effect of warming the polar 
region and cooling the equatorial region, thus reducing the temperature difference $\Delta T$. This can be seen in the temperature deviation plots of Figures 4 and 7, in which the gradient of the temperature deviation is essentially opposite to the imposed differential heating. Model calculations presented here show that smaller values of $\Delta T$ stabilize this large Hadley cell and move the system away from the transitions to multiple convection cells (to the left in Figures 9 and 12). Therefore, the thermal conveyor belt function of a large Hadley cell enhances its persistence. On the other hand, if $\Delta T$ increases (for some other reason) to a value where the single Hadley cell is replaced by multiple cells, this would curtail the thermal conveyor belt acting from equator to pole. See temperature deviation plots of Figures 6 and 8 , in which the gradient of the temperature deviation is essentially in the vertical direction. As a result, the polar regions would cool relative to the equatorial regions, and $\Delta T$ would effectively increase, pushing the system further to the right along the bifurcation curve.

We conjecture that this feedback mechanism, from the convection cell flow back to the temperature difference $\Delta T$, is present in real planetary atmospheres and implies a modification of the predictions of our model. The effect is most easily stated with reference to Figure 3: the interval of bistability in $\Delta T$ would lengthen and the cusp point would move to smaller values of $R$. In other words, the net effect of this thermal feedback would be to increase both the likelihood and the amplitude of the hysteresis behaviour demonstrated in the model.

The results of this paper lead to more questions than answers, and will form the basis of much future work. First, the conjecture stated in the previous paragraph requires further exploration. In particular, the thermal feedback due to the "thermal conveyor belt" may be incorporated into the differential heating term containing $-\Delta T \cos (2 \theta)$. Secondly, the model should be reconsidered to take into account the fact that the atmosphere of the Earth is a strongly stratified compressible fluid, with properties very different from water. The vertical motion of a strongly stratified fluid is inhibited, thus it is expected to cause an elongation (in $\theta$ ) of the cells. The cells in a Boussinesq fluid typically have an aspect ratio close to 1 , which implies that $R$ must be rather large in order to see only 3 cells. It is expected that in a strongly stratified fluid a much smaller $\mathrm{R}$ (for a similar $\Delta T$ ) would be sufficient to see a similar number of cells. Therefore, we predict that the phenomena exhibited in this model could exist in the atmosphere of a planet such as the Earth, but for much smaller aspect ratios $R / r_{a}$. Thus, both the thermal feedback and the stratified fluid properties of real atmospheres, which have been neglected in this simple model, can be expected to amplify rather than inhibit the hysteresis mechanism demonstrated for the model.

On the Earth, the growth of glaciers from the poles causes an increase in albedo there, thus increasing the difference $\Delta T$ and favouring further growth of glaciers. This ice-albedo 
feedback effect could be incorporated in the model using energy-balance equations like those of Budyko [3] or Sellers [30].

Further work could also extend the model to physical dimensions on the scale of a planet such as the Earth. In the opposite direction, it would be useful to perform laboratory experiments in a rotating hemispherical shell of fluid with a uniform density (hence zero buoyancy) and a radial driving force of electrostatic or other nongravitational form, to test these mathematical predictions. Such experiments have already been performed in a thin annular geometry [25]. For this case, the mathematical model can be modified to change the forcing mechanism from thermoconvection to electroconvection as for the thin annulus, see $[28,29]$. Other future goals of this work would be an analysis of the non-axisymmetric bifurcations that would lead to rotating waves, and an analysis of a spherical shell model that breaks the north-south reflectional symmetry or assumes different boundary conditions. Still another direction of research would involve a search of the geological record of the Earth and of the properties of the known planets to find evidence that a global climate hysteresis bifurcation like the one described here may have occurred on an existing planet.

\section{Acknowledgments}

The authors would like to thank Wayne Nagata, Martin Golubitsky and Laurette Tuckerman for helpful discussions.

\section{References}

[1] Andereck, C.D. and Hayot, F. Ordered and Turbulent Patterns in Taylor-Couette Flow. NATO ASI Series Physics 297, Plenum, New York (1992).

[2] Beltrame, P., Travnikov, V., Gellert, M. and Egbers, C. GEOFLOW: simulation of convection in a spherical shell under central force field. Nonli. Processes Geophys. 13, (2006) 413-423.

[3] Budyko, M.I. The effect of solar radiation variations on the climate of the earth. Tellus 21, (1969) 611-619.

[4] Chandrasekhar, S. Hydrodynamic and Hydromagnetic Stability. Dover Publications, New York (1981) (first published 1961, Oxford University Press)

[5] Chossat, Pascal. Bifurcation and stability of convective flows in a rotating or not rotating spherical shell. SIAM J. Appl. Math. 37, (1979) 624-647.

[6] Chossat, P. and Iooss, G. The Couette-Taylor Problem. Springer-Verlag, New York (1994).

[7] Govaerts, Willy J.F. Numerical Methods for Bifurcations of Dynamical Equilibria. SIAM, Philadelphia (2000).

[8] Golubitsky, M. and Langford, W.F. Pattern formation and bistability in flows between counterrotating cylinders. Physica D 32, (1988) 362-392. 
[9] Golubitsky, M. and Schaeffer, D.G. Singularities and Groups in Bifurcation Theory Appl. Math. Sci. 51, Springer-Verlag, New York (1985).

[10] Hadley, George. Concerning the cause of the general trade winds. Phil. Trans. 29, (1735) 58-62.

[11] Hart, J.E., Glatzmaier, G.A. and Toomre J. Space-laboratory and numerical simulations of thermal convection in a rotating hemispherical shell with radial gravity. J. Fluid Mech. 173, (1986) 519-544.

[12] Hide, R. and Mason, P.J. Sloping convection in a rotating fluid. Adv. Geophys. 24, (1975) 47-100.

[13] Hignett, P., White, A.A., Carter, R.D., Jackson, W.D.N. and Small, R.M. A comparison of laboratory measurements and numerical simulations of baroclinic wave flows in a rotating cylindrical annulus. Quart. J. Roy. Meteorol. Soc. 111, (1985) 131-154.

[14] Juel, A., Darbyshire, A.G. and Mullin T. The effect of noise on pitchfork and Hopf bifurcations. Proc. Roy. Soc. Lond. A 453, (1997) 2627-2647.

[15] Kuznetsov, Y.A. Elements of Applied Bifurcation Theory. Third Edition. Springer-Verlag, New York (2004).

[16] Langford, W.F., Tagg, R., Kostelich, E.J., Swinney, H.L. and Golubitsky, M. Primary instabilities and criticality in flow between counter-rotating cylinders. Phys. Fluids 31, (1988) 776-785.

[17] Lehoucq, R., Sorensen, D.C. and Yang, C. ARPACK Users' Guide: Solution of Large-Scale Eigenvalue Problems with Implicitly Restarted Arnoldi Methods. SIAM, Philadelphia (1998).

[18] Lesueur, V., Abouelainine, A., Mangeney, A. and Drossart, P. Geostrophic motions of a Boussinesq fluid in a thick rotating spherical shell. Geophys. Astrophys. Fluid Dyn. 91, (1999) 1-43.

[19] Lewis, G.M. and Nagata, W. Linear stability analysis for the differentially heated rotating annulus. Geophys. and Astrophys. Fluid Dyn. 98, (2004) 129-152.

[20] Lewis, G.M. and Nagata, W. Double Hopf bifurcations in the differentially heated rotating annulus. SIAM J. Appl. Math. 63, (2003) 1029-1055.

[21] Li, L., Zhang, P., Liao, X. and Zhang, K. Multiplicity of nonlinear thermal convection in a spherical shell. Phys. Review E 71, (2005)

[22] Lorenz, Edward N. The Nature and Theory of the General Circulation of the Atmosphere. World Meteorological Society (1967)

[23] Marcus, P.S. and Tuckerman L.S. Simulation of flow between concentric rotating spheres. Part 1. Steady states. J. Fluid Mech. 185, (1987) 1-30.

[24] Marcus, P.S. and Tuckerman L.S. Simulation of flow between concentric rotating spheres. Part 2. Transitions. J. Fluid Mech. 185, (1987) 31-65.

[25] Morris, S.W., de Bruyn, J.R. and May, A.D. Patterns at the onset of electroconvection in freely suspended smectic films. J. Stat. Phys. 64, (1991) 1025-1043.

[26] Peixoto, J.P. and Oort, A.H. Physics of Climate. American Institute of Physics, New York (1992).

[27] Rucklidge, A.M. and Champneys, A.R. Boundary effects and the onset of Taylor vortices. Physica D 191, (2004) 282-296.

[28] Rusu, D.D. and Langford W.F. Bistability of vortex modes in annular thermoconvection. In Buescu J. et al. eds., Trends in Mathematics: Bifurcations, Symmetry and Patterns. Birkhäuser Verlag, Basel (2003) 87-99. 
[29] Rusu, D.D. and Langford W.F. Linear stability and pattern formation in annular electroconvection. Accepted to appear in Dynamics of Continuous, Discrete and Impulsive Systems, (2006) 18 Latex pages.

[30] Sellers, W.D. A global climate model based on the energy balance of the Earth-atmosphere system. $J$. Appl. Meteorol. 8, (1969) 392-400.

[31] Williams, G.P. Baroclinic annulus waves. J. Fluid Mech. 49, (1971) 417-449.

[32] Yanagisawa, T. and Yamagishi, Y. Rayley-Bénard convection in a spherical shell with infinite Prandtl number at high Rayleigh number. J. Earth Sim. 4, (2005) 11-17. 